\title{
Linguistic Versus Latent Relations for Modeling Coherent Flow in Paragraphs
}

\author{
Dongyeop Kang Hiroaki Hayashi Alan W Black Eduard Hovy \\ Carnegie Mellon University, Pittsburgh, PA, USA \\ \{dongyeok, hiroakih, awb, hovy\}@cs.cmu.edu
}

\begin{abstract}
Generating a long, coherent text such as a paragraph requires a high-level control of different levels of relations between sentences (e.g., tense, coreference). We call such a logical connection between sentences as a (paragraph) flow. In order to produce a coherent flow of text, we explore two forms of intersentential relations in a paragraph: one is a human-created linguistical relation that forms a structure (e.g., discourse tree) and the other is a relation from latent representation learned from the sentences themselves. Our two proposed models incorporate each form of relations into document-level language models: the former is a supervised model that jointly learns a language model as well as discourse relation prediction, and the latter is an unsupervised model that is hierarchically conditioned by a recurrent neural network (RNN) over the latent information. Our proposed models with both forms of relations outperform the baselines in partially conditioned paragraph generation task. Our codes and data are publicly available ${ }^{1}$.
\end{abstract}

\section{Introduction}

When composing multiple sentences into a paragraph, as in novels or academic papers, we often make design decisions in advance (Byrne, 1979) such as topic introduction and content ordering to ensure better coherence of the text. For instance, McKeown (1985); Swan (2002) proposed effective patterns for scientific writing: a hypothesis at first, followed by supporting sentences to validate the hypothesis, and lastly a concluding sentence. We call such a logical connection between sentences in a written paragraph as a flow. A coherent flow between sentences requires an understanding of various factors including tense, coreference, plans (Appelt, 1982; Hovy, 1991), scripts

\footnotetext{
${ }^{1}$ https://github.com/dykang/flownet
}

(Tomkins, 1978) and several others. We focus on the paragraph-level plan between sentences.

In text planning, underlying relations in text are broadly categorized into two forms: an explicit human-defined relation (e.g., a discourse tree) (Reiter and Dale, 2000) or an implicitly learned latent relation (Yang et al., 2016). While the former is defined and manually annotated based on linguistic theories, the latter is simply determinable from how people in fact put sentences together. In this work, we provide an empirical comparison between a linguistically-informed and a latent form of relations in context of a paragraph generation.

We compare the effectiveness of the two forms of relations using language modeling for paragraph generation. Due to the different characteristics of the two forms, we employ comparable but different components in addition to the base language model. For linguistic relations (e.g., discourse), we cast the problem into multi-task learning of supervised language modeling and discourse relation prediction. On the other hand, for latent relations, we learn an unsupervised hierarchical language model that is hierarchically conditioned by RNNs over linear operations between sentences.

We evaluate our models on partial paragraph generation task; producing the rest of text in a paragraph given some context of text. We observe that linguistically annotated discourse relations help produce more coherent text than the latent relations, followed by other baselines.

\section{Related Work}

There has been a variety of NLG systems that incorporate additional information between sentences (Appelt, 1982; Reiter and Dale, 2000; Gatt and Krahmer, 2018) which can be broadly categorized into two forms: linguistic and latent. 
Linguistic relations are explicitly represented as external labels in the form of predefined rules or plans, formats, knowledge base, discourse parses, and more. Hovy (1985, 1990); Dalianis and Hovy (1996) integrated text planning in generation, where the plans are considered in knowledge, formatted rules and so forth. However, they are limited to small scale (i.e. few examples) and hand-written rules. Kang et al. (2017); Gardent et al. (2017); Kang et al. (2018b); Wang et al. (2018) used an external knowledge base to micro-planning for generating a corresponding text, while our work focuses on comparing two forms of relations from the text itself.

Moore and Paris (1993); Young and Moore (1994) utilized discourse structures such as rhetorical structure theory (RST) (Mann and Thompson, 1988) for parsing a document. A script (Tomkins, 1978) is another structured representation that describes a typical sequence of events in a particular context. Zhang et al. (2016); Ji and Eisenstein (2014) proposed better discourse parsers using neural networks. The prior works, however, used the discourse representations to describe the structure of the paragraph, while we focus on applicability of the discourse relations to language generation.

Latent relations use implicit information in a document such as hierarchical structure of the document: Lin et al. (2015); Chung et al. (2016) used hierarchical RNN for modeling a document. Similarly, the hierarchical model can be extended to other variants such as attention (Yang et al., 2016), encoder-decoder framework (Serban et al., 2017; Sordoni et al., 2015), auto-encoding ( $\mathrm{Li}$ et al., 2015), and multiscale (Chung et al., 2016). However, the hierarchical recurrence of sentences, which is dependent on topics, are less likely modeling a flow of a document.

We further summarize the fundamental differences between the two forms of relations in Appendix.

\section{FlowNet: Language Modeling with Inter-sentential Relations}

We propose language models that incorporate each relation to capture a high-level flow of text.

\subsection{Discourse-driven FlowNet}

As a linguistic relation, we employ RST (Mann and Thompson, 1988) trees to represent discourse connections in the text. For simplicity, we limit usage of the discourse trees by only considering relations between adjacent phrases $^{2}$ : relations are inserted between adjacent phrases and represented as a flattened sequence of phrases and relations. If two consecutive RST relations are given, the deeper level of relation is chosen. If the central elementary discourse unit (EDU) or phrase is after its dependent, the relation is excluded. We consider each sequence of the flattened discourse relations as a writing flow. For example, people often write a text by elaborating basic information (Elaboration) and then describing a following statement attributed to the information (Attribution).

We view discourse relations as additional labels to predict at the same time we predict next words in language modeling. Specifically, we propose to jointly train a model that predicts a sequence of words and a sequence of RST labels by taking advantage of shared representations, following previous sequence labeling problems such as named entity recognition (Collobert et al., 2011) and partof-speech tagging (Huang et al., 2015). Note that the RST relations are only used during training to obtain better representation for the two tasks, but not at test time.

Figure 1(a) shows our FlowNet using discourse relations. Let a paragraph be a sequence of sentences $D=\left\{s_{1}, s_{2}, \ldots, s_{M}\right\}$. This model treats adjacent sentences as pairs for learning the standard seq2seq model. The first objective is to maximize the likelihood of the current sentence given the previous sentence. Hence, we maximize the following:

$$
\mathbb{L}_{s 2 s}=\sum_{j} \log P\left(w_{i j} \mid w_{i,<j}, s_{i-1}\right)
$$

where $s_{i}=\left\{w_{i 1}, w_{i 2}, \ldots, w_{i T_{i}}\right\}$, and $T_{i}$ is the number of tokens of $s_{i}$.

To better guide the model with discourse context, we use the shared representations to predict RST relations at the same time. For each paragraph, we run the pre-trained RST parser (Ji and Eisenstein, 2014) and flatten the parse tree to obtain RST relations for each sentence $Y_{i}=\left(y_{1}, \ldots, y_{K_{i}}\right)$, where $K_{i}$ is the number of discourse relations in $s_{i}$. We then make a label sequence over tokens in the sentence with

\footnotetext{
${ }^{2}$ The full discourse tree can be incorporated using other types of language model such as Tai et al. (2015).
} 


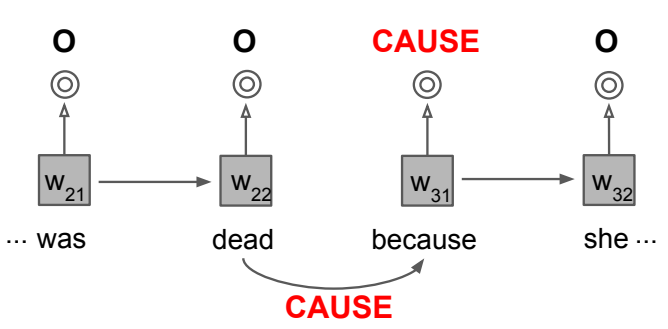

(a) Discourse-driven

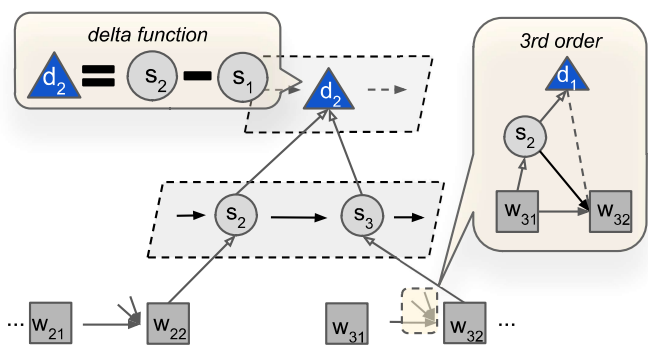

(b) Delta-driven

Figure 1: FlowNet with linguistic (i.e., discourse) versus latent (i.e., delta) relation. (a) For each word, a form of discourse relation and next word are jointly predicted using CRF (๑) and language model, respectively. (b) Decoding $w_{i}$ is conditioned on previous word $\left(w_{i-1}\right)$, previous sentence $\left(s_{i-1}\right)$, and delta between two previous sentences $\left(d_{i-2}\right)$. Best viewed in color.

by placing $y$ at the first word of EDUs and filling up the rest with a null relation $o: Y_{i}^{\prime}=$ $\left(o, \ldots, o, \mathbf{y}_{\mathbf{1}}, o, \ldots, \mathbf{y}_{\mathbf{K}_{\mathbf{i}}}, o, \ldots, o\right)$. We incorporate a sequence labeling objective by employing conditional random field (Lafferty et al., 2001) to find the label sequence that maximizes the score function for each sentence $s_{i}: \mathbf{S}\left(s_{i}, Y_{i}^{\prime}\right)=$ $\sum_{j=1}^{T_{i}-1} W_{y_{j}^{\prime}, y_{j+1}^{\prime}}^{T} h_{j}+b_{y_{j}^{\prime}, y_{j+1}^{\prime}}$ where $h_{j}, W$ and $b$ are the hidden representation of $w_{i j}$, weight matrix, and the bias vector corresponding to the pair of labels $\left(y_{i}^{\prime}, y_{i+1}^{\prime}\right)$, respectively. For training, we maximize the conditional likelihood:

$$
\mathbb{L}_{C R F}=\mathbf{S}\left(s_{i}, y_{i}^{\prime}\right)-\sum_{\mathbf{y} \in \mathbb{Y}_{x}} \log \mathbf{S}\left(s_{i}, \mathbf{y}\right)
$$

where $\mathbb{Y}_{x}$ represents all possible discourse label sequences. Decoding is done by greedily predicting the output sequence with maximum score. Both training and decoding can be computed using dynamic programming. The final objective is represented as the sum of two objective functions:

$$
\mathbb{L}_{\text {disc }}=\mathbb{L}_{s 2 s}+\alpha * \mathbb{L}_{C R F}
$$

where $\alpha$ is a scaling parameter to control the impact of CRF objective. The value is chosen empirically by searching based on validation set.

\subsection{Delta-driven FlowNet}

In this model, we aim to utilize latent representations to characterize the flow between sentences. Specifically we define delta, subtractions of hidden represenations of adjacent sentences as such latent information. Figure 1(b) shows how we hierarchically model different levels of information: words, sentences, and deltas.

Each word is encoded using a RNN encoder $g_{\text {word }}$. We take the last hidden representation of word as sentence embeddings $s_{1}, \ldots, s_{M}$. Similar to hierarchical RNN (Lin et al., 2015), each sentence representation is encoded using another RNN encoder $g_{\text {sent }}$. While discourse flow provides an explicit relation symbols, delta flow calculates a latent relation by subtracting previous representation $s_{i-1}$ from current representation $s_{i}{ }^{3}$ :

$$
d\left(s_{i-1}, s_{i}\right)=d_{i-1}=s_{i}-s_{i-1}
$$

Given a sequence of $M-1$ delta relations $d_{1}, \ldots, d_{M-1}$ for a paragraph of $M$ sentences, we again encode them using another RNN encoder $g_{\text {delta }}$. The model takes the word, sentence and delta information altogether to predict the next $(t-$ th) word in the $m$-th sentence:

$$
h_{t}=f\left(h_{t-1}, x_{t}, s_{m-1}, d_{m-2}\right)
$$

where $x_{t}$ is a word representation, $s_{m-1}$ is a sentence representation and $d_{m-2}$ is a delta information. Note that sentence representation is from the previous sentence, and delta information is calculated by two previous sentences. If there is no previous information given, the parameters are randomly initialized.

\section{Experiment}

Due to the absence of goal-oriented language generation task, we collect paragraph data and define a new task of generating partial text of a paragraph given some context.

\subsection{Data}

We collect paragraphs from three different domains: Papers are paragraphs extracted from

\footnotetext{
${ }^{3}$ Our experiment includes a comparison among other types of linear operations between sentences such as addition or a learnable function.
} 


\begin{tabular}{l|rrr}
\hline & Train & Valid & Test \\
\hline Papers & 16,173 & 899 & 899 \\
Scifi & 157,031 & 8,724 & 8,724 \\
Fantasy & 317,654 & 17,649 & 17,649 \\
\hline
\end{tabular}

Table 1: Number of paragraphs in our dataset.

academic manuscripts in computer science domain from the PeerRead (Kang et al., 2018a), and Fantasy and SciFi are paragraphs of two frequent categories extracted from the BookCorpus (Zhu et al., 2015), where paragraphs are extracted using the line breaker in the dataset.

We only use paragraphs whose lengths are from 4 to 7 , in order to measure the performance change according to paragraph length. The dataset is randomly split by $0.9 / 0.05 / 0.05$ for train, valid, and test set, respectively. Table 1 shows the numbers of paragraphs for each domain. All paragraphs are parsed into RST trees using the state-of-the-art discourse parser by Ji and Eisenstein (2014).

\subsection{Bridging: Partial Paragraph Generation}

We evaluate our models on partial text generation task; given a partial information (e.g., some sentences), producing the rest of text.

[1] Inside the club we moved straight for the bar. [2] Devlin ordered a beer for himself and a glass of my favorite wine for me. [3] I love that I didn't have to tell him what I wanted. [4] He knew me well and always thought about what I wanted or needed, in and out of bed.

Figure 2: Bridging task: given [1] and [4] sentences, guessing [2,3] sentences (red, underlined).

Figure 2 shows our bridging task. It requires a generation of masked sentences in the middle of a paragraph given the first and the last sentences. If only the first sentence is given, the generation can be too divergent. The existence of the last sentence makes the generation more coherent and converged to some point.

We evaluate it with one hard and one soft automatic metrics: METEOR (M) (Banerjee and Lavie, 2005) and VectorExtrema (VE) (Liu et al., 2016) by calculating cosine similarity of averaged word embeddings (Pennington et al., 2014), and human performance.

\subsection{Models and Setup}

We compare various baseline seq2seq models which encode the context; a concatenated first and last sentences, and decode the intermediate words: S2S is attentional seq2seq model (Bahdanau et al., 2014), and HS2S: is a hierarchical version of the S2S by combining two baselines: HRNN (Lin et al., 2015) hierarchically models sequence of words and sentences, and HRED (Serban et al., 2017; Sordoni et al., 2015) encodes the given context and decodes the words. FlowNet (delta/disc.) is our proposed language model with delta and discourse relations, respectively.

We find the best hyper-parameters on validation set using grid search. Here are the final parameters used: 32 for batch size, 25 for maximum sentence length, 300 for word embedding size initialized by GloVe (Pennington et al., 2014), 1 LSTM layer (Hochreiter and Schmidhuber, 1997) with 512 size, clipping by $0.25,0.2$ learning rate and 0.5 decay rate with Adagrad (Duchi et al., 2011) optimizer, and 50,000 for the vocabulary size. The total number of distinct discourse relations is 44 .

\subsection{Results}

\begin{tabular}{r|cccccc}
\hline & \multicolumn{2}{|c}{ Papers } & \multicolumn{2}{c}{ SciFi } & \multicolumn{2}{c}{ Fantasy } \\
\hline & $\mathbf{M}$ & $\mathbf{V E}$ & $\mathbf{M}$ & $\mathbf{V E}$ & $\mathbf{M}$ & VE \\
\hline S2S & 3.7 & 56.3 & 3.5 & 71.0 & 3.3 & 66.3 \\
HS2S & 3.7 & 54.7 & 3.4 & $\mathbf{7 3 . 0}$ & 3.0 & 69.7 \\
\hline FlowNet (delta) & 3.1 & $\mathbf{5 8 . 5}$ & 3.6 & 69.7 & 3.6 & $\mathbf{7 3 . 9}$ \\
FlowNet (disc.) & $\mathbf{4 . 0}$ & 57.2 & $\mathbf{4 . 2}$ & 70.3 & $\mathbf{3 . 9}$ & 71.8 \\
\hline
\end{tabular}

Table 2: Performance on bridging task. METEOR and VectorExtrema are used. The higher the better.

In Table 2, both discourse and delta driven FlowNet outperform the baseline models across most of the metrics except for VecterExtrema on Scifi. Especially, as the number of training size increases (Papers $<<\mathrm{SCiF} i<$ Fantasy), the improvements gained from the FlowNet become bigger. This is probably because the model learns more information of the (discourse or latent) relations from the larger data.

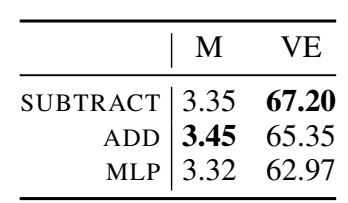

Table 3: Comparison of different delta functions.

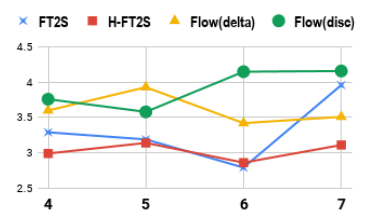

Figure 3: Comparison of paragraph lengths. Best viewed in color.
Table 3 shows performance comparison among different delta operations: SUBTRACT, ADD, and 
FIRST: Satyrs never wear armor, including helmets, Newel began, using his hands expressively.

LAST: Anyhow, as we actors were laying siege, a big chunk of the battlement dislodged from atop the tower.

REF: [M1] "But years ago I was in a play, and the helm was part of my costume. [M2] During the big battle scene, a few of us were assailing a castle. [M3] We had quite a set. [M4] The main tower must have been fifteen feet tall, fashioned from real stone. HUMAN: [M1] Actually he needed to wear any protectors to prevent him from a big accident. [M2] We planned to make a prank cam to make him wear those always. [M3] "I have a good idea," Newel kept talking continuously. [M4] "Let's play a role like we are under the attack.

S2S: [M1] he's a good man [M2] the UNK, the one who's a man who's a man and the other [M3] and the other, the one who 's a good friend [M4] he's a good man

HS2S: [M1] i'm not sure that," he said [M2] i'm not sure that i'm not sure [M3] i'm not sure that i'm not a fool [M4] "i'm not sure that," he said

FLOWNET (DELTA): [M1] he's a good man [M2] i'm not sure what to do [M3] i'm not sure that i'm not going to be a vampire [M4] he's a good man

FLOWNET (DISC.): [M1] perhaps they were not quite good, but he was not a master, and they were the most powerful [M2] the only way to do not like a little, but i' d been in the world [M3] "you're right," he said "i am not a fool you're here [M4] you're going to be a bit more than the other

Table 4: An example paragraph and predicted texts in Fantasy dataset. Given FIRST and LAST sentences, the models generate middle sentences (e.g., [M1] $\rightarrow$ [M2]..). REF and HUMAN are reference middle sentences and sentences written by human annotator, respectively. Please find more examples in the appendix.

MLP which is a multi-layer perceptron network. All scores are macro-averaged across datasets. While ADD shows good performance on METEOR, SUBTRACT does on the soft metric (i.e., VecExt), indicating that subtraction can help the model capture the better semantics than the other functions. Figure 3 shows how performance changes on Fantasy as the paragraph lengths increase. Both of FlowNet achieve more improvements when generating longer paragraphs. Especially, discourse relations achieve the best performance at length 6 and 7 .

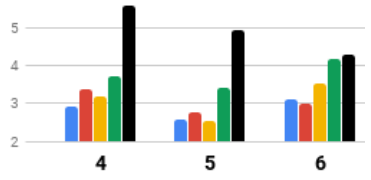

(a) By paragraph lengths

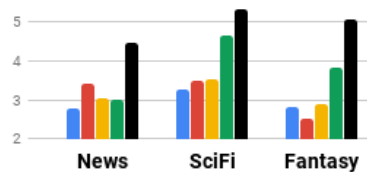

(b) By domains
Figure 4: Comparison (METEOR) with human performance (black bars): S2S (blue), HS2S (red), Flow:delta (yellow), and Flow:disc. (green). Best viewed in color.

We conduct a comparison with human performance (See Figure 4). We randomly choose 100 samples per dataset and per paragraph length and ask an annotator to perform the bridging task on the final 1,000 samples. Human outperforms the models by large margins. FlowNet with discourse relations outperforms the FlowNet with latent relations and other baselines by a large margin. As the paragraph length increases or more data is trained, discourse relations become more useful.

Table 4 shows an example paragraph with text produced by the models as well as reference and human annotation. Given only the partial context (i.e., first and last sentences), bridging task is very challenging even for human. The reference sentences and human annotations are semantically very different indeed. Among the latent models, FlowNet (delta) produces more coherent flow of text compared to S2S and HS2S. Surprisingly, FlowNet (discourse) enables generating more diverse sentences with a bit of coherence, because each sentence is generated based on the representation conditioned on the predicted RST discourse relation.

\section{Conclusion and Discussion}

We explore two forms of inter-sentential relations: linguistic relation such as discourse relations and a latent representation learned from the text. The proposed models for both relations achieve significant improvements over the baselines on partial paragraph generation task.

Despite the empirical effectiveness and difference between the linguistic and latent relations, they are not directly aligned for comparison. A potential direction for future study is to directly couple them together and see whether one form contains the other, or vice versa. Another direction is to check their effectiveness on top of the recent pre-trained language models.

\section{Acknowledgements}

We also thank Jason Weston, Dan Jurafsky, and anonymous reviewers for their helpful comments. 


\section{References}

Douglas E Appelt. 1982. Planning natural-language utterances to satisfy multiple goals. Technical report, SRI INTERNATIONAL MENLO PARK CA ARTIFICIAL INTELLIGENCE CENTER.

Dzmitry Bahdanau, Kyunghyun Cho, and Yoshua Bengio. 2014. Neural machine translation by jointly learning to align and translate. arXiv preprint arXiv:1409.0473.

Satanjeev Banerjee and Alon Lavie. 2005. Meteor: An automatic metric for $\mathrm{mt}$ evaluation with improved correlation with human judgments. In Proceedings of the acl workshop on intrinsic and extrinsic evaluation measures for machine translation and/or summarization, pages 65-72.

Donn Byrne. 1979. Teaching writing skills. Longman.

Junyoung Chung, Sungjin Ahn, and Yoshua Bengio. 2016. Hierarchical multiscale recurrent neural networks. CoRR, abs/1609.01704.

Ronan Collobert, Jason Weston, Léon Bottou, Michael Karlen, Koray Kavukcuoglu, and Pavel Kuksa. 2011. Natural language processing (almost) from scratch. Journal of Machine Learning Research, 12(Aug):2493-2537.

Hercules Dalianis and Eduard Hovy. 1996. Aggregation in natural language generation. In Trends in Natural Language Generation An Artificial Intelligence Perspective, pages 88-105. Springer.

John Duchi, Elad Hazan, and Yoram Singer. 2011. Adaptive subgradient methods for online learning and stochastic optimization. Journal of Machine Learning Research, 12(Jul):2121-2159.

Claire Gardent, Anastasia Shimorina, Shashi Narayan, and Laura Perez-Beltrachini. 2017. Creating training corpora for nlg micro-planning. In 55th annual meeting of the Association for Computational Linguistics (ACL).

Albert Gatt and Emiel Krahmer. 2018. Survey of the state of the art in natural language generation: Core tasks, applications and evaluation. Journal of Artificial Intelligence Research, 61:65-170.

Sepp Hochreiter and Jürgen Schmidhuber. 1997. Long short-term memory. Neural Computation, 9:17351780 .

Eduard H. Hovy. 1985. Integrating text planning and production in generation. In IJCAI.

Eduard H Hovy. 1990. Pragmatics and natural language generation. Artificial Intelligence, 43(2):153197.

Eduard H Hovy. 1991. Approaches to the planning of coherent text. In Natural language generation in artificial intelligence and computational linguistics, pages 83-102. Springer.
Zhiheng Huang, Wei Xu, and Kai Yu. 2015. Bidirectional lstm-crf models for sequence tagging. CoRR, abs/1508.01991.

Yangfeng Ji and Jacob Eisenstein. 2014. Representation learning for text-level discourse parsing. In Proceedings of the 52nd Annual Meeting of the Association for Computational Linguistics (Volume 1: Long Papers), volume 1, pages 13-24.

Dongyeop Kang, Waleed Ammar, Bhavana Dalvi, Madeleine van Zuylen, Sebastian Kohlmeier, Eduard Hovy, and Roy Schwartz. 2018a. A dataset of peer reviews (peerread): Collection, insights and nlp applications. In Proceedings of NAACL-HLT.

Dongyeop Kang, Varun Gangal, Ang Lu, Zheng Chen, and Eduard Hovy. 2017. Detecting and explaining causes from text for a time series event. In Conference on Empirical Methods on Natural Language Processing.

Dongyeop Kang, Tushar Khot, Ashish Sabharwal, and Eduard Hovy. 2018b. Adventure: Adversarial training for textual entailment with knowledge-guided examples. In The 56th Annual Meeting of the Association for Computational Linguistics (ACL), Melbourne, Australia.

John D. Lafferty, Andrew McCallum, and Fernando Pereira. 2001. Conditional random fields: Probabilistic models for segmenting and labeling sequence data. In $I C M L$.

Jiwei Li, Minh-Thang Luong, and Daniel Jurafsky. 2015. A hierarchical neural autoencoder for paragraphs and documents. In $A C L$.

Rui Lin, Shujie Liu, Muyun Yang, Mu Li, Ming Zhou, and Sheng Li. 2015. Hierarchical recurrent neural network for document modeling. In Proceedings of the 2015 Conference on Empirical Methods in Natural Language Processing, pages 899-907.

Chia-Wei Liu, Ryan Lowe, Iulian V Serban, Michael Noseworthy, Laurent Charlin, and Joelle Pineau. 2016. How not to evaluate your dialogue system: An empirical study of unsupervised evaluation metrics for dialogue response generation. arXiv preprint arXiv:1603.08023.

William C Mann and Sandra A Thompson. 1988. Rhetorical structure theory: Toward a functional theory of text organization. Text-Interdisciplinary Journal for the Study of Discourse, 8(3):243-281.

Kathleen R McKeown. 1985. Discourse strategies for generating natural-language text. Artificial Intelligence, 27(1):1-41.

Johanna D Moore and Cécile L Paris. 1993. Planning text for advisory dialogues: Capturing intentional and rhetorical information. Computational linguistics, 19(4):651-694. 
Jeffrey Pennington, Richard Socher, and Christopher Manning. 2014. Glove: Global vectors for word representation. In Proceedings of the 2014 conference on empirical methods in natural language processing (EMNLP), pages 1532-1543.

Ehud Reiter and Robert Dale. 2000. Building natural language generation systems. Cambridge university press.

Iulian Vlad Serban, Alessandro Sordoni, Ryan Lowe, Laurent Charlin, Joelle Pineau, Aaron C Courville, and Yoshua Bengio. 2017. A hierarchical latent variable encoder-decoder model for generating dialogues. In AAAI, pages 3295-3301.

Alessandro Sordoni, Yoshua Bengio, Hossein Vahabi, Christina Lioma, Jakob Grue Simonsen, and JianYun Nie. 2015. A hierarchical recurrent encoderdecoder for generative context-aware query suggestion. In Proceedings of the 24th ACM International on Conference on Information and Knowledge Management, pages 553-562. ACM.

Judith A. Swan. 2002. The science of scientific writing. In Book.

Kai Sheng Tai, Richard Socher, and Christopher D Manning. 2015. Improved semantic representations from tree-structured long short-term memory networks. arXiv preprint arXiv:1503.00075.

Silvan S Tomkins. 1978. Script theory: Differential magnification of affects. In Nebraska symposium on motivation. University of Nebraska Press.

Qingyun Wang, Xiaoman Pan, Lifu Huang, Boliang Zhang, Zhiying Jiang, Heng Ji, and Kevin Knight. 2018. Describing a knowledge base. In CoRR, volume abs/1809.01797.

Zichao Yang, Diyi Yang, Chris Dyer, Xiaodong He, Alex Smola, and Eduard Hovy. 2016. Hierarchical attention networks for document classification. In Proceedings of the 2016 Conference of the North American Chapter of the Association for Computational Linguistics: Human Language Technologies.

R Michael Young and Johanna D Moore. 1994. Dpocl: A principled approach to discourse planning. In Proceedings of the Seventh International Workshop on Natural Language Generation, pages 13-20. Association for Computational Linguistics.

Biao Zhang, Deyi Xiong, Jinsong Su, Qun Liu, Rongrong Ji, Hong Duan, and Min Zhang. 2016. Variational neural discourse relation recognizer. In EMNLP.

Yukun Zhu, Ryan Kiros, Richard S. Zemel, Ruslan Salakhutdinov, Raquel Urtasun, Antonio Torralba, and Sanja Fidler. 2015. Aligning books and movies: Towards story-like visual explanations by watching movies and reading books. 2015 IEEE International Conference on Computer Vision (ICCV), pages 1927. 